Review

\title{
Photodynamic therapy in oral potentially malignant disorders-Critical literature review of existing protocols
}

\author{
Jéssica Araújo Figueira $^{\mathrm{a}, *}$, Vanessa Cristina Veltrini ${ }^{\mathrm{b}}$ \\ a Oral Oncology Center and Department of Pathology and Clinical Propedeutics, Araçatuba Dental School, Univ Estadual Paulista-UNESP, São Paulo, Brazil \\ b Oral Pathology, Dentistry Department, Maringá State University-UEM, Paraná, Brazil
}

\section{A R T I C L E I N F O}

\section{Keywords:}

Clinical protocols

Oral cancer

Oral dysplasia

Oral medicine

Oral potentially malignant disorders

Oral premalignant lesions

Photodynamic therapy

\begin{abstract}
A B S T R A C T
Introduction: Oral cancer is a serious public health issue. Apart from its high rate of prevalence, incidence and mortality, it can often result in more complex and expensive treatment when diagnosed late. Potentially malignant disorders (PMDs) can precede oral cancer, and are usually treated by surgical excision. However, in many cases patients are elderly and multiple interventions may be required. Photodynamic therapy (PDT) is a simple alternative, which has been successfully used in the treatment of oral PMDs.

Objective: Due to the lack of standardization regarding photosensitizers (PTSs), types of irradiation, and methods of application, the objective of this study was to analyze existing PDT protocols in an attempt to identify the one that demonstrates greater efficiency, reliability and feasibility in the treatment of oral PMDs for both researchers and clinicians.

Methods: Original clinical studies published only in English between 1993 and 2016 were searched in Pubmed/ Medline database using the following keywords: photodynamic therapy; oral potentially malignant disorder; oral premalignant lesions. Review articles; experimental studies; case-reports; commentaries; and letters to the Editor were excluded from the selection.

Results and conclusion: Based on the 16 studies selected, the topical 5-ALA-20\% PTS, associated to a LED light applied for 15 min with a 7-day interval between sessions emerged as the most frequently used PDT protocol, with satisfactory results. Due to its low rate of side effects, this PDT protocol presents good potential for the treatment of oral PMDs. Further clinical studies are required to ascertain its long-term validity in preventing oral cancer.
\end{abstract}

\section{Introduction}

Potentially malignant disorders (PMDs) refer to benign, but morphologically altered tissues, which present a greater risk of undergoing malignant transformations [1]. The World Health Organization (WHO), in 2005, changed the previous terminology "potentially malignant lesions" and "potentially malignant conditions" to PMD [2].

The term dysplasia is characterized by the presence ofysplasia is characterized by the presence of abnormal epithelial architecture, disordered cell growth, changes in the nuclear-cytoplasmic ratio, atypical mitosis, and other alterations. Dysplasia can be classified into mild, moderate, and intense, according to subjective microscopic analysis [3]. It is believed that the more severe the epithelial dysplasia, the greater the risk of malignization [3].

Oral PMD management still requires clearer definition. Surgical excision, laser surgery, and cryotherapy associated with the reduction of risk factors, as well as follow up examinations for different periods of time have been suggested $[2,4,5]$. Photodynamic therapy (PDT) has recently emerged as a potential alternative in the treatment of PMDs, since it is capable of promoting total or partial regression of these conditions with few side effects $[2,4,5]$.

\subsection{Photodynamic therapy}

PDT is a minimally invasive treatment, successfully applied in head and neck PMDs and malignant disorders. The technique is simple and can usually be performed on an outpatient basis $[4,5]$.

A topical or systemic photosensitizer (PTS) drug is administered, which selectively binds to atypical cells. After an incubation period, a source of light of suitable wavelength is applied on the target tissue. It promotes the absorption of photons by the PTS, which turns into an extremely unstable molecule [6]. This molecule, while searching for stability, transfers the light energy to the oxygen in the cellular environment. This reaction generates oxygen reactive species, which has a

\footnotetext{
* Corresponding author at: Silva Jardim, 593, casa B, Jardim Sumaré, 16015-450, Araçatuba, São Paulo, Brazil.

E-mail address: jessica.a.figueira@gmail.com (J.A. Figueira).
} 
cytotoxic effect on the tissue, leading to cell death [6].

When compared to more invasive techniques, PDT systemic effects are insignificant, it is minimally toxic to normal tissue, and presents reduced morbidity and excellent aesthetic results. It can be applied in association with any other conventional treatment and repeated as often as necessary, without generating cumulative toxicity [4-6]. Sensitivity, pain, swelling, burning sensation, taste alterations, ulcerations, and loss of local sensation have been reported with PDT, but often with a low magnitude $[7,8]$.

\subsection{Photosensitizer}

Photosensitizers (PTSs) are drugs that promote the transfer of light energy to the cellular environment, resulting in the formation of highly reactive chemical species, which act in the destruction of target cells.

PTSs tend to accumulate in atypical cells, but the mechanisms responsible for this process are not completely understood [6]. Several hypotheses have been proposed. One of them concerns the predominance of blood vessels with morphological changes in tumors as a result of neovascularization and the lack of lymphatic drainage, creating greater permeability and drug retention [6]. Another hypothesis states that some drugs preferentially bind to low-density-lipoprotein (LDL) receptors, which are over-expressed in atypical cells [6]. There is also the perception that low $\mathrm{pH}$ found in atypical cells cytoplasm promotes drug ionization, making it more hydrophilic, increasing PTS retention inside the cell [9].

To select the appropriate PTS for each clinical situation, features such as toxicity, selectivity, wavelength necessary for its activation, effectiveness, side effects, route of administration and cost have to be taken into consideration.

There are three generations of drugs, according to their chronological order of development. The first generation includes hematoporphyrin derivative (HpD) and porfimer sodium (Photofrin ${ }^{\circ}$ ) [6]. To improve some of drawbacks presented by the first generation of PTSs (relatively low absorption and skin photosensitivity), the second generation of PTSs was developed, with excellent results [6]. It includes 5aminolevulinic acid (5-ALA); hypericin; phthalocyanine; benzoporphyrin derivatives, such as Verteporfin ; and meta tetrahydroxyphenyl chlorin (mTHPC) derivatives, such as Foscan ${ }^{\circ}$ [6]. A new, third generation of PTSs is now being developed to improve atypical cell selectivity, and represents an area of active research [6].

\subsubsection{Light sources}

The application of light in an appropriate wavelength (600-800 nm), also known as the "therapeutic window", excites the PTS, generating a reaction with the oxygen present in the cells. Shorter wavelengths have low tissue penetration, while irradiation with longer wavelengths may not have enough power to generate reactive oxygen [6]. The blue light $(450-495 \mathrm{~nm})$, for example, penetrates less efficiently through the tissue when compared to the red $(620-750 \mathrm{~nm})$ and infrared light ( $>750 \mathrm{~nm}$ ), which can penetrate deeper in the tissue [6].

The choice of a light source is dependent on the lesion (tissue feature, size, location and accessibility), type of PTS (spectrum of absorption and administration), cost and availability of light systems [6].

Theoretically, any light source may be used in PDT [10]. Conventional lamps were the first to be used. Because they have a wide range of wavelengths, filters are required to obtain the desired wavelength [10]. With the introduction of lasers, the use of conventional lamps in PDT has decreased considerably [11]. Laser produces monochromatic wavelengths, which allows easy calculation of light dosimetry and the ideal wavelength for a specific PTS [11]. The light-emitting diode (LED) is also an alternative source of light that has been increasingly used [6].

\subsubsection{Photochemistry and photophysics}

The basic principle of PDT is the formation of cytotoxic agents from the interaction of the oxygen within the cells and the PTS excited by light of an appropriate wavelength.

After activation by light photons, the PTS changes from its primary state $\left({ }^{1} \mathrm{PTS}\right)$ to a singlet excited-state $\left({ }^{1} \mathrm{PTS} *\right)$ [12]. The excited PTS is very unstable and tends to return to its original and energetically more favorable state, which takes place through the emission of heat or fluorescence [12]. In this particular case, photodynamic reactions with therapeutic outcomes do not occur, but have the potential to be used as a diagnostic tool [12]. Another possibility is the passage of ${ }^{1} \mathrm{PTS} *$ to another excitation state, less unstable, called triplet state $\left({ }^{3} \mathrm{PTS}^{*}\right)$, which is capable of promoting two different photodynamic reactions [12]:

Type I reaction - PTS in its excited triplet state $\left({ }^{3} \mathrm{PTS} *\right)$ interacts with cellular substrates $[6,12]$. Through the transfer of electrons, ions are generated. These react with molecular oxygen, making reactive oxygen species (ROS), such as superoxide, hydrogen peroxide and hydroxyl radicals $[6,12]$.

Type II reaction - Direct transfer of energy between ${ }^{3}$ PTS $^{*}$ and molecular oxygen occurs, exciting $\mathrm{O}_{2}$ and leading to the formation of singlet oxygen $\left({ }^{1} \mathrm{O} 2\right)$, which is also a ROS $[6,12]$.

Reactive oxygen species are highly reactive free radicals, which have the ability to interact with different molecules and damage their normal function [13]. This is a key factor for PDT cytotoxicity [13]. ROS formation can lead to tumor destruction through three main biological mechanisms: 1) direct destruction by activating cell death pathways, such as apoptosis, necrosis and autophagy; 2) damage to the tumor vasculature, by suppressing the delivery of oxygen and nutrients; and 3) stimulation of inflammatory and/or immune response [13].

\section{Methodology}

Original clinical studies, published only in English between 1993 and 2016 were searched in Pubmed/Medline database using different combinations of the following keywords: photodynamic therapy; oral potentially malignant disorder; oral premalignant lesion. Sixteen clinical studies using PDT to treat oral PMD were selected. The objective was to compare the PDT protocols used and their respective results. Review articles, experimental studies (in vitro or animal studies), casereports, commentaries, and letters to the Editor were excluded from the selection.

\section{Results}

The selected articles are displayed in Table 1.

Based on the selected studies, a comparative analysis of the protocols and their respective results was performed. Absence or incomplete data were disregarded.

Methodological difficulties were found during table organization. In Kübler et al. [16], Tsai et al. [17], Rigual et al. [22] and Shafirstein et al. [8], for example, the number of sessions, time of light activation and interval among sessions were absent. In Yu et al. [21], Lin et al. [23], Pietruska et al. [24] the type of laser was not described, and in Fan et al. [15], Tsai et al. [17], Pietruska et al. [24] and Maloth et al. [26] the number of recurrences was not disclosed.

Number of sessions ranged from 1 to 10; light application time from 10 to $16.6 \mathrm{~min}$; and the interval among sessions ranged from 3.5 to 14 days, with the 7-day interval being the most frequent $[4,7,14-16]$. The time from PTS administration and light activation was between 48 and $96 \mathrm{~h}$ when used intravenously; $1.5-4 \mathrm{~h}$ when applied topically; $0.5-4 \mathrm{~h}$ orally; and $1.5 \mathrm{~h}$ for intralesional application [4,7,14-16].

Light dose administered ranged from 50 to $200 \mathrm{~J} / \mathrm{cm}^{2}$. The most frequent was $100 \mathrm{~J} / \mathrm{cm}^{2}$, used in 10 of the 16 studies $[7,8,16-21,23,25]$. In four studies, the light dose administered was variable [4,14,15,22]. Grant [14] used 50-100 J/ $\mathrm{cm}^{2}$; Fan et al. [15], $100-200 \mathrm{~J} / \mathrm{cm}^{2}$; Rigual et al. [22], 50-75 J/ $\mathrm{cm}^{2}$ and Jerjes et al. [4] used $100-200 \mathrm{~J} / \mathrm{cm}^{2}[4,14,15,22]$.

Regarding the PTS, the most frequently used drug was the 5- 


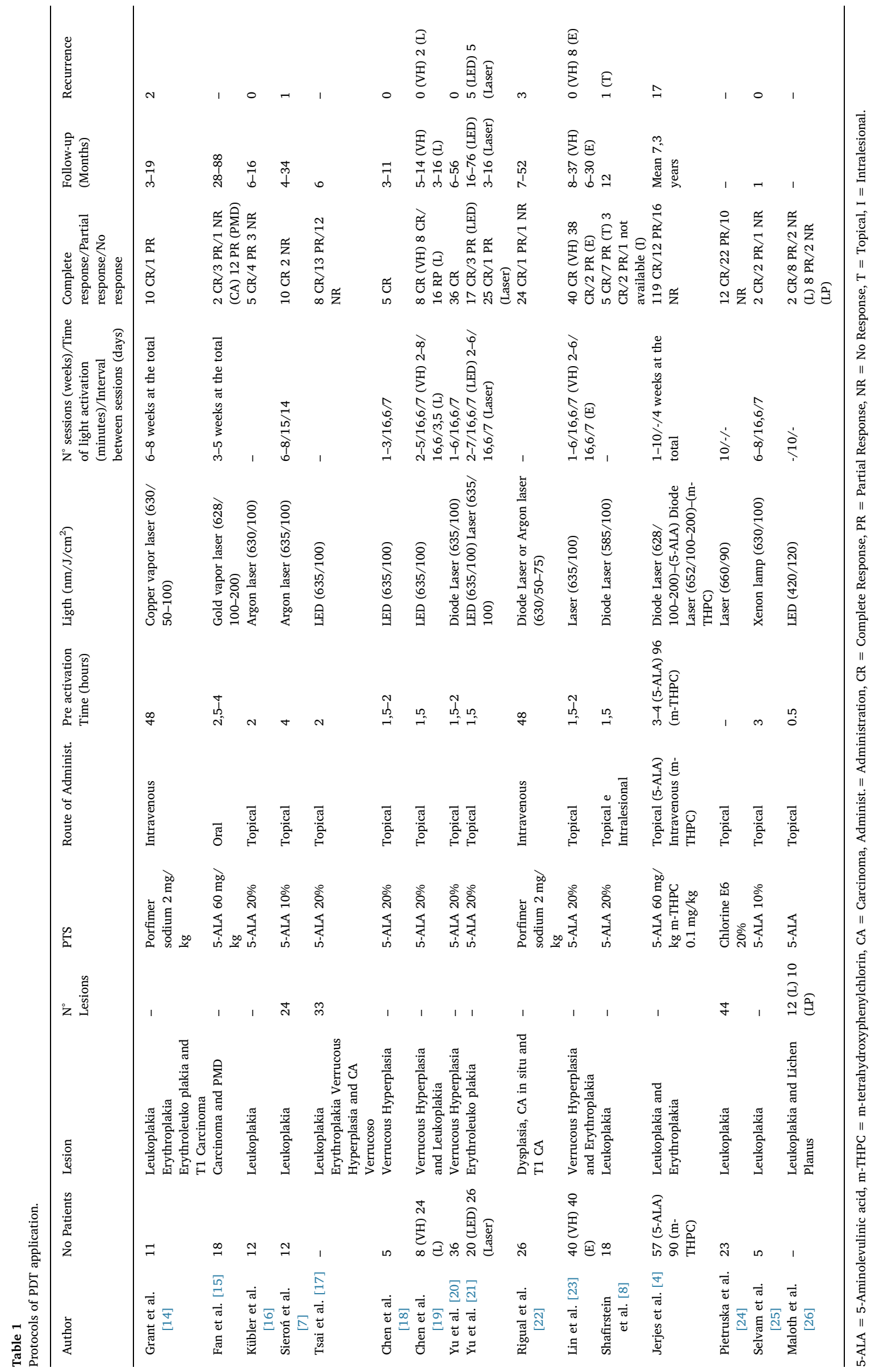


aminolevulinic acid (5-ALA) $20 \%$ (Levulan ${ }^{\circ}$ ), applied topically in 8 of the 16 studies $[8,16,17-21,23]$. The 5-ALA was applied in a total of 256 patients, with complete remission in $78 \%$ of the lesions, partial remission in $18 \%$ and no remission in $6 \%$. The recurrence rate was $7 \%$.

\subsection{Discussion}

The 5-aminolevulinic acid (5-ALA) is a prodrug that undergoes biotransformation and only becomes active in vivo. It quickly accumulates in large quantities in tissues [27]. This feature is one of the major advantages of 5-ALA compared to other PTSs, and may explain why it has been more frequently used [27]. With 5-ALA, side effects tend to be reduced, since its clearance is faster than other PTSs [28]. It is used topically in the treatment of surface lesions, such as oral PMD, and is capable of penetrating 1-2 mm into the tissues [29].

LED ( 5 studies) and diode laser (4 studies) were the most frequently used light sources $[4,8,19-22,26]$. One study using 5-ALA compared LED and Laser in oral PMD, finding no significant differences between the light sources [21]. According to the authors, LED was simpler, lighter, smaller, more portable, and cheaper with the potential to replace Laser in the future. However, the authors call attention to its durability, which may be compromised by chip impairment as a result of the heat generated [21]. The Laser source (specification not provided), on the other hand, was considered more stable, more durable and was equipped with adjustable power, although it was heavier and more expensive [21]. The authors concluded that when used in erythroleukoplakia, the choice of the light source would depend on the budget [21].

No explanation is available for the existence of varied responses using the same PDT protocol. Probably the results are influenced by specific features of PMDs such as size, color, presence of atypia and thickness of the keratin layer. Best results were achieved in minor lesions and with thinner keratin layers [8,20,21,23,25]. For example, a study reported better clinical outcome for dysplastic erythroleukoplakia lesions than for non-dysplastic oral lesions such as leukoplakia lesions [21]. The histological, biological and structural characteristics of erythroleukoplakia contribute to the successful clinical outcome of these lesions. Compared to non-dysplastic oral lesions, dysplastic erythroleukoplakia lesions have less keratotic epithelial surface, as well as thinner and more permeable epithelium. Thus, the topical PTS may more easily diffuse into these dysplastic epithelia, resulting in good absorption, while the reducing effect of the thinner keratin layer on light intensity was minimal [21].

The measures adopted in case of recurrence were the repetition of the PDT protocol, lesion excision or just follow-up, depending on the lesion or patient's wish $[4,14,21-23]$.

PDT side effects are uncommon. Mild to moderate pain and local edema were the most reported effects $[4,7,8,14,15,17,20,22,23]$. Skin photosensitivity, burning sensation and discomfort were also common $[4,8,15,16,20,22-24,25]$. Although not frequent, erythema, ulceration and secondary infection were also present $[7,8,16,17]$. For pain control, nonsteroidal anti-inflammatory drugs (NSAIDs) and opiates were considered sufficient $[4,5]$. Systemic PTS resulted in some important side effects, when compared to topical PTS, such as residual systemic photosensitization, which can last for several days or weeks, edema, sunburn and superficial skin necrosis, when skin is exposed to bright light [4].

PDT consists of an effective, non-toxic strategy in the treatment of oral PMDs. It is a minimally invasive technique that results in less morbidity and mutilation to the oral tissues, and can be applied several times, with minimal side effects. However, standardized protocols, clinical trials with larger samples, as well as long term follow-ups are still required.

\section{Conclusion}

PMDs are a therapeutic challenge for dental surgeons. Based on the short-term clinical applicability and the benefits demonstrated, PDT can be a useful treatment strategy for the management of oral PMDs. Based on the literature review conducted, the protocol using 5-ALA-20\% topical PTS in association with a LED light source, applied for $15 \mathrm{~min}$ with 7-day intervals between sessions, the number of sessions depending on the response to the treatment, emerged as a strong candidate in the treatment of PMDs. The use of systemic sensitizer (Porfimer sodium $2 \mathrm{mg} / \mathrm{kg}$ ) yielded an initial complete response rate of $90 \%$, which surely would also warrant its inclusion in any anticipated clinical trials. However, due to the side effects resulting from its use, and the small size of oral lesions compared to skin lesions, topical use seems to be best indicated in oral PMDs. It should be noted, however, that the primary aim of PDT is to prevent invasive malignancy occurring. Invasive tumors often arise in apparently normal areas of the mucosa so that it cannot be anticipated that the resolution of obvious oral PMDs will actually avoid the development of cancer. Further clinical studies with long term follow up are required to determine not only the best protocol, but also the ultimate benefits of PDT in the treatment of oral PMDs.

\section{References}

[1] World Health Organization, Report of a Meeting of Investigations on the Histological Classification of Precancerous Lesions, World Health Organization, Geneva, 1973.

[2] I. Van der Waal, Potentially malignant disorders of the oral and oropharyngeal mucosa; terminology, classification and present concepts of management, Oral Oncol. 45 (2009) 317-323.

[3] T. Izumo, Oral premalignant lesions: from the pathological viewpoint, Int. J. Clin. Oncol. 6 (1) (2011) 15-26.

[4] W. Jerjes, T. Upile, Z. Hamdoon, C.A. Mosse, S. Akram, C. Hopper, Photodynamic therapy outcome for oral dysplasia, Lasers Surg. Med. 43 (2011) 192-199.

[5] W. Jerjes, Z. Hamdoon, C. Hopper, Photodynamic therapy in the management of potentially malignant and malignant oral disorders, Head Neck Oncol. 4 (2012) 16.

[6] P. Agostinis, K. Berg, K.A. Cengel, T.H. Foster, A.W. Girotti, S.O. Gollnick, S.M. Hahn, M.R. Hamblin, A. Juzeniene, D. Kessel, M. Korbelik, J. Moan, P. Mroz, D. Nowis, J. Piette, B.C. Wilson, J. Golab, Photodynamic therapy of cancer: an update, CA. Cancer J. Clin. 61 (2011) 250-281.

[7] A. Sieroń, M. Adamek, A. Kawczyk-Krupka, S. Mazur, L. Ilewicz, Photodynamic therapy (PDT) using topically applied delta-aminolevulinic acid (ALA) for the treatment of oral leukoplakia, J. Oral Pathol. Med. 32 (2003) 330-336.

[8] G. Shafirstein, A. Friedman, E. Siegel, M. Moreno, W. Bäumler, C.Y. Fan, K. Morehead, E. Vural, B.C. Stack Jr, J.Y. Suen, Using 5-aminolevulinic acid and pulsed dye laser for photodynamic treatment of oral leukoplakia, Arch. Otolaryngol. Head Neck Surg. 137 (2011) 1117-1123.

[9] R. Pottier, J.C. Kennedy, The possible role of ionic species in selective biodistribution of photochemotherapeutic agents toward neoplastic tissue, J. Photochem. Photobiol. B 8 (1990) 1-16.

[10] Z. Huang, A review of progress in clinical photodynamic therapy, Technol. Cancer Res. Treat. 4 (2005) 283-293.

[11] M.A. Calin, S.V. Parasca, Light sources for photodynamic inactivation of bacteria, Lasers Med. Sci. 24 (2009) 453-460.

[12] Z. Luksiene, Photodynamic therapy: mechanism of action and ways to improve the efficiency of treatment, Medicina (Kaunas) 39 (2003) 1137-1150.

[13] A.P. Castano, P. Mroz, M.R. Hamblin, Photodynamic therapy and anti-tumour immunity, Nat. Rev. Cancer 6 (2006) 535-545.

[14] W.E. Grant, C. Hopper, P.M. Speight, A.J. Macrobert, S.G. Bown, Photodynamic therapy of malignant and premalignant lesions in patients with 'field cancerization' of the oral cavity, J. Laryngol. Otol. 107 (1993) 1140-1145.

[15] K.F. Fan, C. Hopper, P.M. Speight, G. Buonaccorsi, A.J. MacRobert, S.G. Bown, Photodynamic therapy using 5-aminolevulinic acid for premalignant and malignant lesions of the oral cavity, Cancer 78 (1996) 1374-1383.

[16] A. Kübler, T. Haase, M. Rheinwald, T. Barth, J. Mühling, Treatment of oral leukoplakia by topical application of 5-aminolevulinic acid, Int. J. Oral Maxillofac. Surg. 27 (1998) 466-469.

[17] J.C. Tsai, C.P. Chiang, H.M. Chen, S.B. Huang, C.W. Wang, M.I. Lee, Y.C. Hsu, C.T. Chen, T. Tsai, Photodynamic Therapy of oral dysplasia with topical 5-aminolevulinic acid and light-emitting diode array, Lasers Surg. Med. 34 (2004) 18-24.

[18] H.M. Chen, C.T. Chen, H. Yang, M.Y. Kuo, Y.S. Kuo, W.H. Lan, Y.P. Wang, T. Tsai, C.P. Chiang, Successful treatment of oral verrucous hyperplasia with topical 5aminolevulinic acid-mediated photodynamic therapy, Oral Oncol. 40 (July) (2004) 630-637.

[19] H.M. Chen, C.H. Yu, P.C. Tu, C.Y. Yeh, T. Tsai, C.P. Chiang, Successful treatment of oral verrucous hyperplasia and oral leukoplakia with topical 5-aminolevulinic acidmediated photodynamic therapy, Lasers Surg. Med. 37 (2005) 114-122. 
[20] C.H. Yu, H.M. Chen, H.Y. Hung, S.J. Cheng, T. Tsai, C.P. Chiang, Photodynamic therapy outcome for oral verrucous hyperplasia depends on the clinical appearance, size, color, epithelial dysplasia, and surface keratin thickness of the lesion, Oral Oncol. 44 (June) (2008) 595-600.

[21] C.H. Yu, H.P. Lin, H.M. Chen, H. Yang, Y.P. Wang, C.P. Chiang, Comparison of clinical outcomes of oral erythroleukoplakia treated with photodynamic therapy using either light-emitting diode or laser light, Lasers Surg. Med. 41 (2009) 628-633.

[22] N.R. Rigual, K. Thankappan, M. Cooper, M.A. Sullivan, T. Dougherty, S.R. Popat, T.R. Loree, M.A. Biel, B. Henderson, Photodynamic therapy for head and neck dysplasia and cancer, Arch. Otolaryngol. Head Neck Surg. 135 (2009) 784-788.

[23] H.P. Lin, H.M. Chen, C.H. Yu, H. Yang, Y.P. Wang, C.P. Chiang, Topical photodynamic therapy is very effective for oral verrucous hyperplasia and oral erythroleukoplakia, J. Oral Pathol. Med. 39 (2010) 624-630.

[24] M. Pietruska, S. Sobaniec, P. Bernaczyk, M. Cholewa, J.K. Pietruski, E. Dolińska,
A. Skurska, E. Duraj, G. Tokajuk, Clinical evaluation of photodynamic therapy efficacy in the treatment of oral leukoplakia, Photodiagn. Photodyn. Ther. 11 (2014) $34-40$.

[25] N.P. Selvam, J. Sadaksharam, G. Singaravelu, R. Ramu, Treatment of oral leukoplakia with photodynamic therapy: a pilot study, J. Cancer Res. Ther. 11 (2015) 464-467.

[26] K.N. Maloth, N. Velpula, S. Kodangal, et al., Photodynamic therapy - a non-invasive treatment modality for precancerous lesions, J. Lasers Med. Sci. 7 (1) (2016) 30-36.

[27] M.L. Davila, Photodynamic therapy, Gastrointest. Endosc. Clin. N. Am. 21 (2011) 67-79.

[28] M. Triesscheijn, P. Baas, J.H. Schellens, F.A. Stewart, Photodynamic therapy in oncology, Oncologist 11 (2006) 1034-1044.

[29] R.R. Allison, Photodynamic therapy: oncologic horizons, Future Oncol. 10 (2014) 123-124. 